\title{
RAREFACTION OF LIQUIDS IN A SPHERICAL SHELL DUE TO LOCAL RADIAL LOADS WITH APPLICATION TO BRAIN DAMAGE*
}

\author{
CHAI-CHIN SUH, WEN-JEI YANG and J. H. MCELHANEY \\ Department of Mechanical Engineering, The University of Michigan, Ann Arbor. Michigan \\ 48104 , U.S.A.
}

\begin{abstract}
An experimental study is conducted to investigate the rarefaction of untreated liquids in a spherical glass container due to a local, radial ultrasonic disturbance of sinusoidal. square pulse, or impulsive nature. Water and dilute polymer solution are used. The effects of the loading pattern and liquid properties on the location and magnitude of the maximum negative pressure in the fluid are examined. The possible locations of brain damage are indicated on the basis of the experimental results. No evidence which produces a so-called contrecoup lesion is found, however.
\end{abstract}

\section{INTRODUCTION}

IN RECENT years, considerable attention has been focused on the mechanism of brain damage resulting from impact. Two major hypotheses have been proposed to explain the mechanism: the rotation hypothesis and the cavitation hypothesis. The former hypothesizes that shear strains resulting from skull rotation rather than skull translation are responsible for the irreversible rupturing of the brain tissue. On the other hand, the cavitation hypothesis explains that the capillary rupturing at the pole opposite to the point of impact-contrecoup lesion-is caused by tensile stresses (reduced pressure) which are produced at the site during impact.

Goldsmith (1966) has presented a thorough review of previously employed theoretical and experimental methods describing the formation of brain trauma and head injury. Engin (1969) by Laplace transform technique and Benedict et al. (1970) by finite difference technique have theoretically studied the dynamic response of a fluid-filled elastic spherical shell subjected to a local radial impact load. Engin's results indicate the brain damage to occur at the points of rarefaction of the fluid: at location $0.57 R$ from the opposite pole and $0.45 R$ from the pole both on the impact line, where $R$ is the inner radius of the spherical shell. $\mathrm{He}$ also predicts the possible locations on the skull susceptible to severe damage at (i) the pole where the skull is in the state of bending, (ii) the circular region around the pole with the angle of $35^{\circ}$ where tensile stresses develop repeatedly, and (iii) the opposite pole where high tensile stresses are generated after the reflection of both the solid and the fluid waves one after the other. The results of Benedict et al. (1970) demonstrate that two focal points of redueed pressure occur within the fluid at the impact pole immediately under the load and at the counter pole in a very diffuse area. Both studies are confirmed by the experimental work of Unterharnscheidt and Sellier (1966) on the mechanics and pathomorphology of traumatic brain injuries. The latter demonstrate that brain damage at the impact pole is highly localized and a surface phenomenon, whereas that at the counter pole is more diffuse and deeper in inward penetration. All these studies support the cavitation hypothesis by demonstrating the existence of the regions of reduced pressure.

The paper reports the experimental measurements of the pressure pattern in a fluidfilled transparent spherical vessel subjected

\footnotetext{
*Received 23 August 1971.
} 
to a local radial ultrasonic load. Untreated water and dilute polymer solution are used, since the physical properties of the brain resemble those of a fluid and, in particular, the intercranial-fluid shows some resemblance to water (Goldsmith, 1966). The polymer solution is prepared to possess the viscosity of the brain. The possible location of brain damage is indicated on the basis of the experimental data.

\section{EXPERIMENTAL APPARATUS}

Figure 1 shows the schematic diagram of the experimental apparatus. This unit consists of a function generator, a power supply system, a converter, a horn, a spherical container, a dual-beam oscilloscope, dual-trace amplifier and a hydrophone. A sinusoidal voltage signal of $20 \mathrm{kHz}$ generated by a Hewett Packard Model 203A variable phase function generator was amplified to $900 \mathrm{~V}$ (peak to peak) in a custom made h.f., highvoltage power amplifier. This amplified signal was the input to the converter which produces a corresponding sinusoidal strain of the same frequency. Efficiency of conversion was 95 per cent. This strain in turn was amplified by the exponential portion of the horn. The amplification factor is equal to the ratio of the diameter of the upper portion of the horn to that of the tip of the exponential portion of the horn. The power input was varied by adjusting the function generator and the power supply system. The motion of the horn tip produces pressure waves.

A 3-1. capacity Pyrex glass spherical container was used to hold the test fluid. Holes in the container at $0,45,90,135$ and $180^{\circ}$ provided the necessary openings for the ultrasonic horn and the hydrophone. A Clevite type $\mathrm{CH}-4 \mathrm{C}-50$ hydrophone was used to measure pressure distribution in the liquid. Its specifications are available in Suh (1971).

The converter and the exponential horn were fastened together with a steel core screw. Care had to be taken to eliminate the air gap between two surfaces by applying silicon paste before tightening the two together so that the acoustic energy was transferred without any loss. The converter is Branson J-type and the exponential horn was made of stainless steel. The unit was fixed to the flange by a snap ring and O-ring which provides necessary sealing. The horn used in the study has resonant frequency of $20 \mathrm{kHz}$.

The container was secured in position between two aluminum plates $1 / 4$ in. thick which are spaced by four adjustable bars as

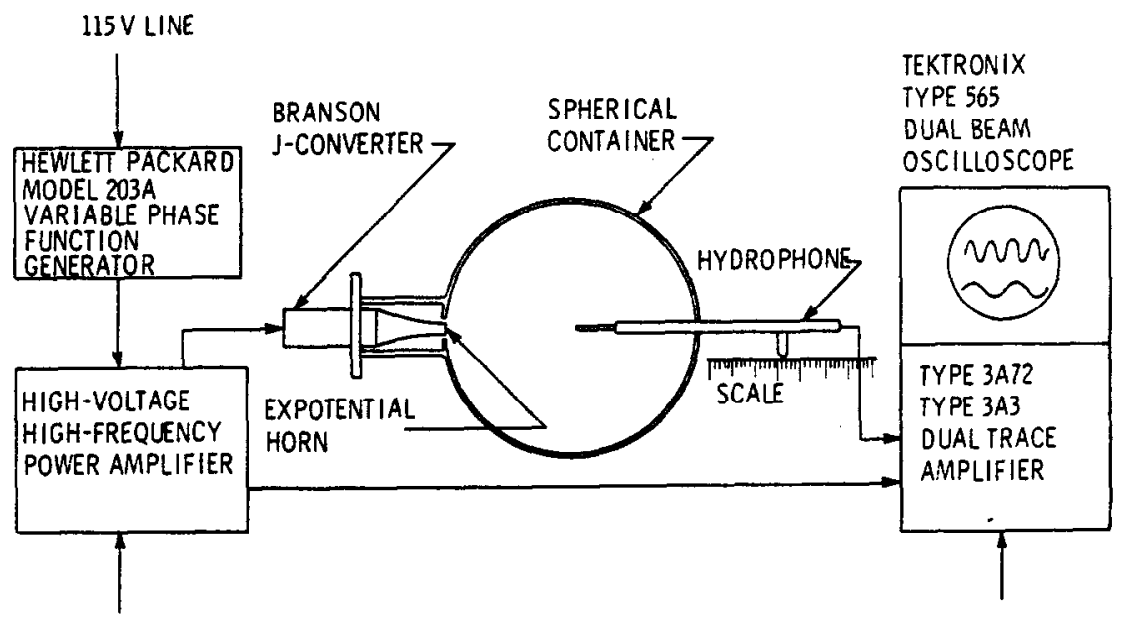

Fig. 1. Schematic diagram of experimental apparatus. 
shown in Fig. 2. Leakage from the test container was prevented by providing necessary sealing as shown in the figure.

Tektronix type 565 dual beam oscilloscope and type $3 \mathrm{~A} 72$ and type $3 \mathrm{~A} 3$ dual trace amplifiers were used to record the voltages across the converter and the known resistance of $4.5 \Omega$, and hydrophone output.

\section{EXPERIMENTAL PROCEDURES}

\section{Sinusoidal input}

The signal from the function generator was fed to the power amplifier and after amplification it was converted to the oscillation. For the fixed location of cone angle and radius, the power input to the converter and the output voltage of the hydrophone were recorded by the oscilloscope. The steady sinusoidal outputs were expected. The r.m.s. power input was computed both from the voltage across the line and from the voltage across the known resistance of $4.5 \Omega$. Recorded hydrophone voltage, peak to zero on the oscilloscope was converted into the pressure in psi by using the hydrophone having sensitivity of $-123 \mathrm{~dB}$ ref $1 \mathrm{~V} /$ bar over the frequency range of $0.3-30 \mathrm{kHz}$. The hydrophone tip was traversed radially at 0 , $45,90,135$ and $180^{\circ}$ of cone angle.

\section{Pulse input}

A square pulse signal of predetermined voltage and duration was fed into the h.f., high-voltage amplifier. The signal was converted into mechanical oscillation by means of the converter. The signal was considerably distorted both in the amplifier and the converter.

\section{Onset of cavitation}

Untreated distilled water was used as the test fluid. The solubility of air in water at room temperature $\left(68^{\circ} \mathrm{F}\right)$ is $18.7 \mathrm{~cm}^{3} / \mathrm{l}$. The onset of cavitation was observed by the appearance of visible bubbles. Simultaneously it was observed that the hydrophone registered a high noise level. The maximum negative pressure refers to a spacial maximum negative amplitude of oscillatory output. The same terminology applies to the case of the square pulse input.

The following procedure developed by Connolly and Fox (1954) was used in the measurement of cavitation voltage. The

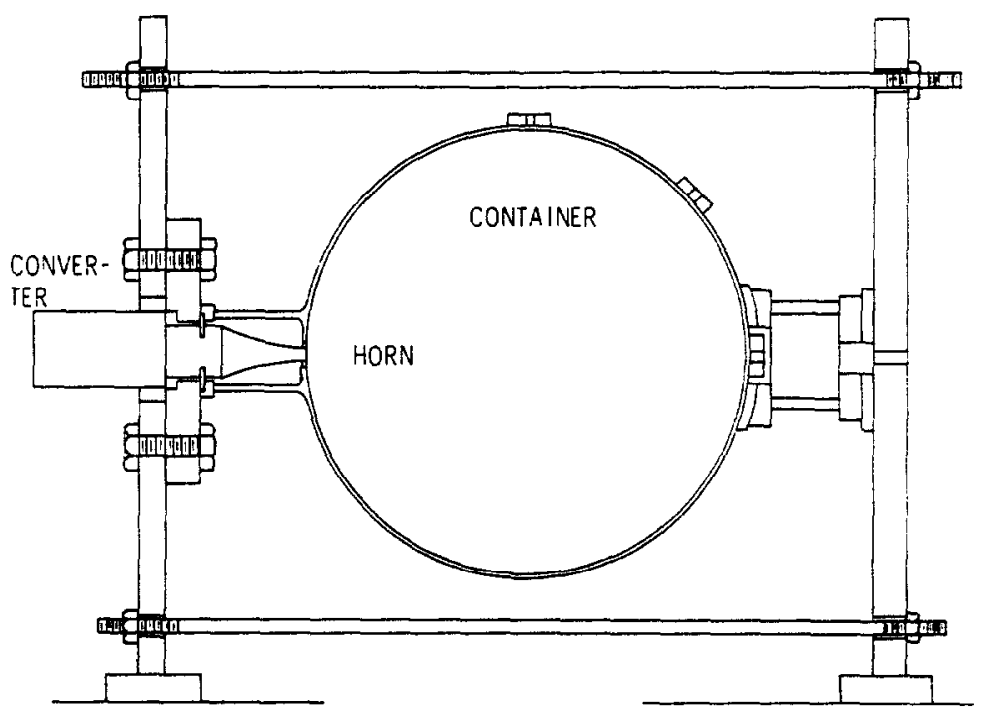

Fig. 2. Schematic diagram of liquid container assembly. 
voltage is increased in small steps and the ultra sound is turned on for a fixed interval, usually $5 \mathrm{sec}$. With $30 \mathrm{sec}$ off, the voltage was increased until cavitation was finally observed. The voltage was then lowered until cavitation ceases. These runs were repeated several times to determine the voltage of the onset of cavitation.

\section{Measurement of horn tip displacement}

A MTI-KD-38 fotonic sensor was used to measure the horn tip displacement. The procedure was as follows.

(1) The sensor was mounted on a fixture in such a way that the axis of the sensor was perpendicular to the horn tip surface and the gap could be adjusted conveniently.

(2) The sensor was adjusted until the panel meter showed its maximum reading. The maximum meter reading was to correspond to a sensor to surface distance of approximately 20 mil.

(3) The V-distance (mil) relationship was determined by adjusting the sensor to surface distance for every mil without driving the converter. Then the distance which fell to the linear portion of the curve was set.

(4) The conversion factor was found to be $20 \mathrm{mil} / \mathrm{V}$ from the mil-V-curve.
(5) The converter was driven with the known voltage input. Measurement was made for the voltage, peak to zero, in terms of mil using the conversion factor.

The calibration curve relating the converter input voltage to the horn tip displacement was thus constructed.

\section{EXPERIMENTAL RESULTS}

\section{Distilled water}

Figure 3 illustrates the pressure distribution detected in distilled water in response to the sinusoidal power input of $0.0181 \mathrm{~W}$ applied to the converter. The converter input voltage is $17.7 \mathrm{~V}$ (r.m.s.) and the horn tip displacement (zero to peak) is 0.015 mil. No bubble was formed under this power input and the signal from the hydrophone remained a neat sine wave. The maximum negative pressure was read from an individual oscilloscope record at each point. The peak maximum negative pressure in the system was about $1.0 \mathrm{psi}$ which was detected near the horn tip. The next peaks detected were 0.73 psi at the dimensionless radius (divided by the inner radius of the vessel) of about 0.775 on the $0^{\circ}$ axis, and $0.71 \mathrm{psi}$ at the dimensionless radius of 0.175 from the center on the $180^{\circ}$ axis. The

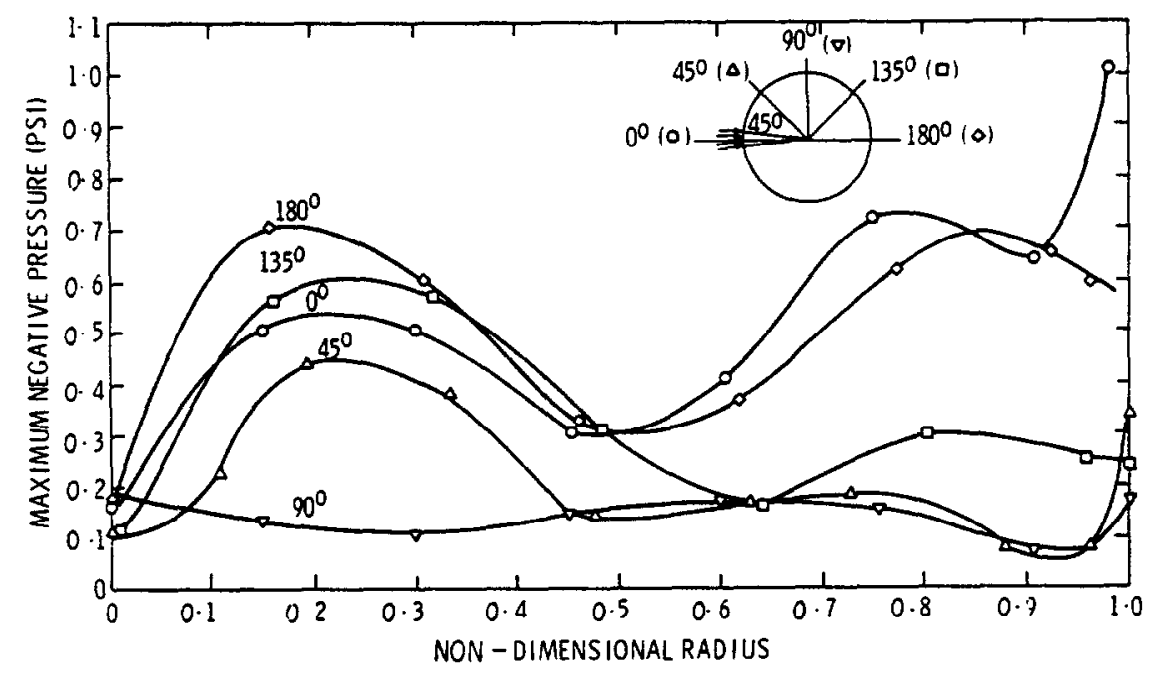

Fig. 3. Measured maximum negative pressure distribution in distilled water subjected to a sinusoidal load over cone angle of $4 \cdot 6^{\circ}$ without cavitation. 
distribution of the maximum negative pressure along the $90^{\circ}$ axis is nearly uniform.

Figure 4 shows the pressure distribution measured in distilled water in response to the sinusoidal power input of $1.05 \mathrm{~W}$ applied to the converter. The converter input voltage was $142 \mathrm{~V}$ (r.m.s.) and the horn-tip displacement was 0.107 mil. At this power input, hissing sounds were heard, and two interesting phenomena were observed: one was the appearance of wildly oscillating signals on the socilloscope screen, and the other was the appearance of a number of bubbles oscillating in the liquid. As the input wattage was increased, the maximum negative pressure shifted its location from the horn tip along the axis toward the opposite pole. When cavitation finally occurred, the location of the maximum negative pressure in the system was detected at the dimensionless radius of 0.32 from the center along the $180^{\circ}$ axis. Its magnitude read $10.4 \mathrm{psi}$. The next maximum was detected at the horn tip with the magnitude of 8.75 psi. Along the load axis, significant pressure fluctuations were observed; however, no significant pressure fluctuations were observed along other axes.

Figure 5 is the measured maximum negative pressure distribution in distilled water in response to a square pulse of $10 \mu \mathrm{sec}$ duration applied to the power amplifier. The horn tip displacement was $0.088 \mathrm{mil}$. Due to the crystal characteristics in the converter, it is questionable whether a single isolated square pulse would appear at the horn tip even if such a pulse were fed into the converter. Hence, this part of the experiment would give only the general trend of response to the pulse-like input. All data points were read from the oscilloscope record of an individual pressure history of a location in response to the square pulse load. The maximum negative pressure occurs after some time has elapsed. The time required to reach the maximum negative pressure varies from point to point: some point may have its maxima when the pulse reaches to the point interested, some point may have its maxima after the pulse passes the point interested. The maximum negative pressure in the system was about 2.25 psi near the horn tip. The next maximum value, approximately $0.8 \mathrm{psi}$ in magnitude was detected at the opposite pole. The maximum negative pressure distributions along other axes in the system appeared to be nearly uniform.

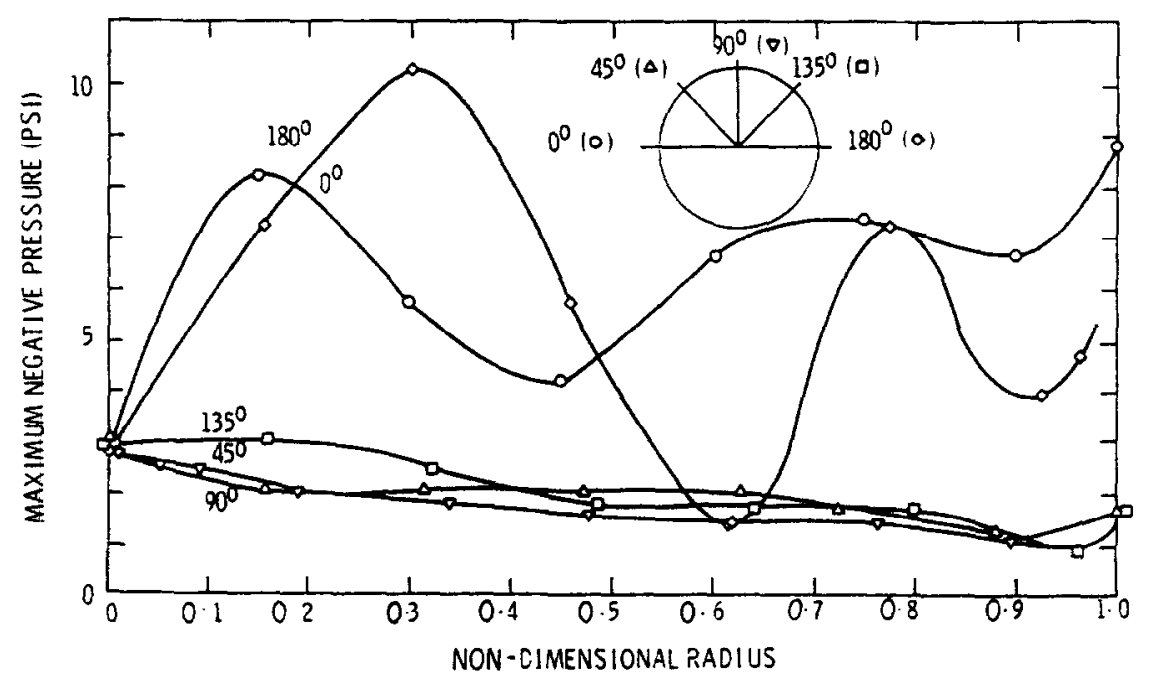

Fig. 4. Measured maximum negative pressure distribution in distilled water subjected to a sinusoidal load over cone angle of $4.6^{\circ}$ with onset of cavitation. 


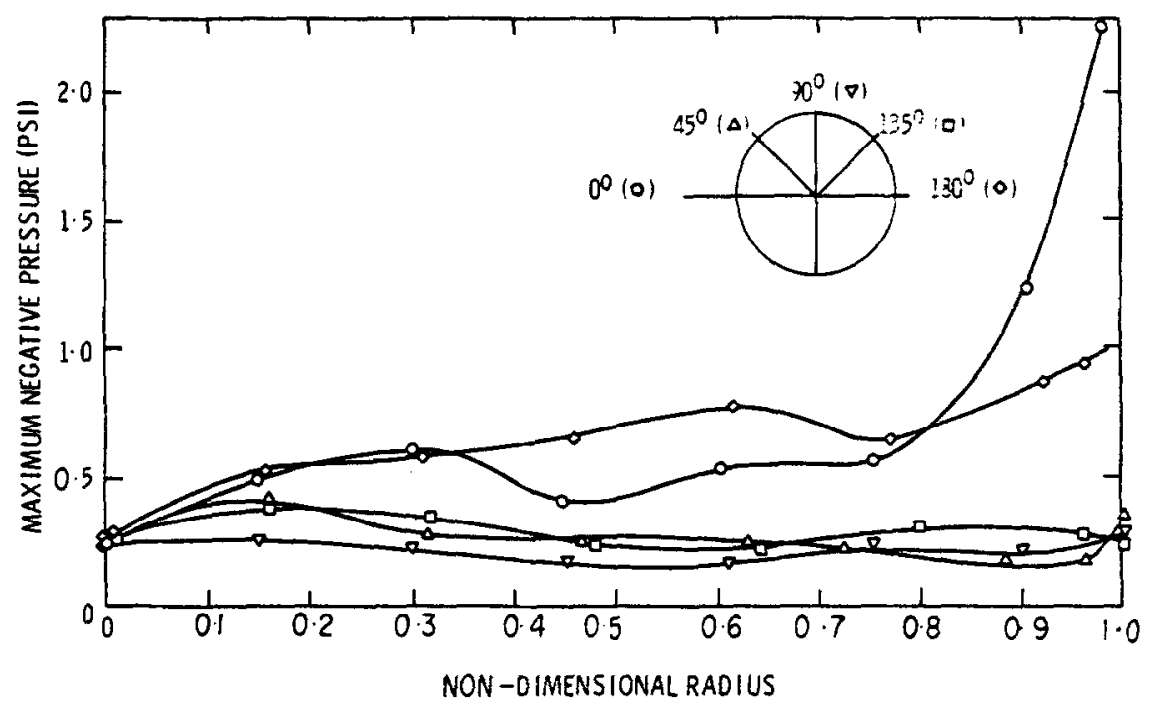

Fig. 5. Measured maximum negative pressure distribution in distilled water subjected to an impulsive load over cone angle of $4.6^{\circ}$.

\section{Dilute solution of SEPARAN AP30}

Similar experiments as above were conducted with a dilute solution of SEPARAN AP30 $(0.05$ per cent by weight of the polymer in distilled water) to investigate the effect of viscosity on the pressure distribution. The solution prepared had the viscosity of $50 \mathrm{cP}$, equivalent to that of brain. The viscosity was measured by a Brookfield Viscometer (Model:
LV, Spindle No. 3, Speed: $60 \mathrm{rev} / \mathrm{min})$. The viscosity of brain varies with investigators depending upon the method of measurement. The value of $50 \mathrm{cP}$ was taken from Stalnaker (1969).

For the case without cavitation, no significant change in the pressure distributions were observed as shown in Fig. 6. The power input to the horn was $0.0174 \mathrm{~W}$ (r.m.s.) and

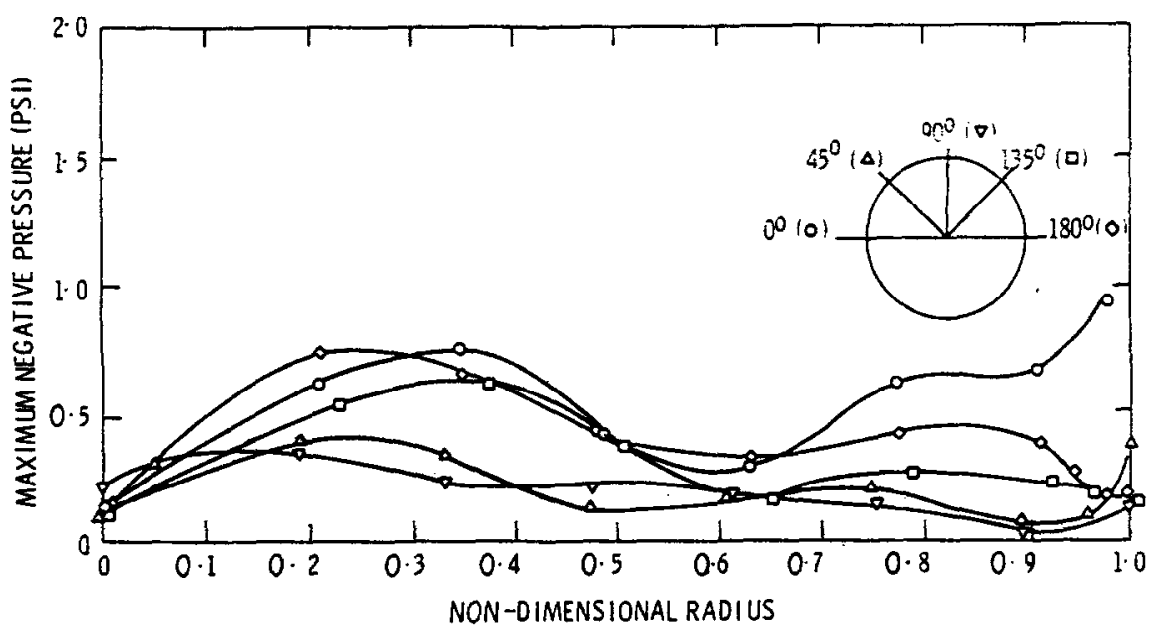

Fig. 6. Measured maximum negative pressure distribution in dilute polymer solution (0.05 per cent by weight of SEPARAN AP30 in distilled water) subjected to a sinusoidal load over cone angle of $4.6^{\circ}$ without cavitation. 
the horn tip displacement was $0.015 \mathrm{mil}$. With the onset of cavitation, more power input ( $4.67 \mathrm{~W}$ r.m.s. and the horn tip displacement of $0.225 \mathrm{mil}$ ) to the solution was required and higher maximum negative pressures as shown in Fig. 7 were obtained. The pressure distribution patterns were quite similar to those of the distilled water except that the highest maximum negative pressure occurred at the horn tip in this case. For the same square pulse input of $10 \mu \mathrm{sec}$ duration applied to the solution, however, lower maximum negative pressure distribution was observed in the entire region of the solution, as shown in Fig. 8. The pressure distribution patterns were similar to those of the distilled

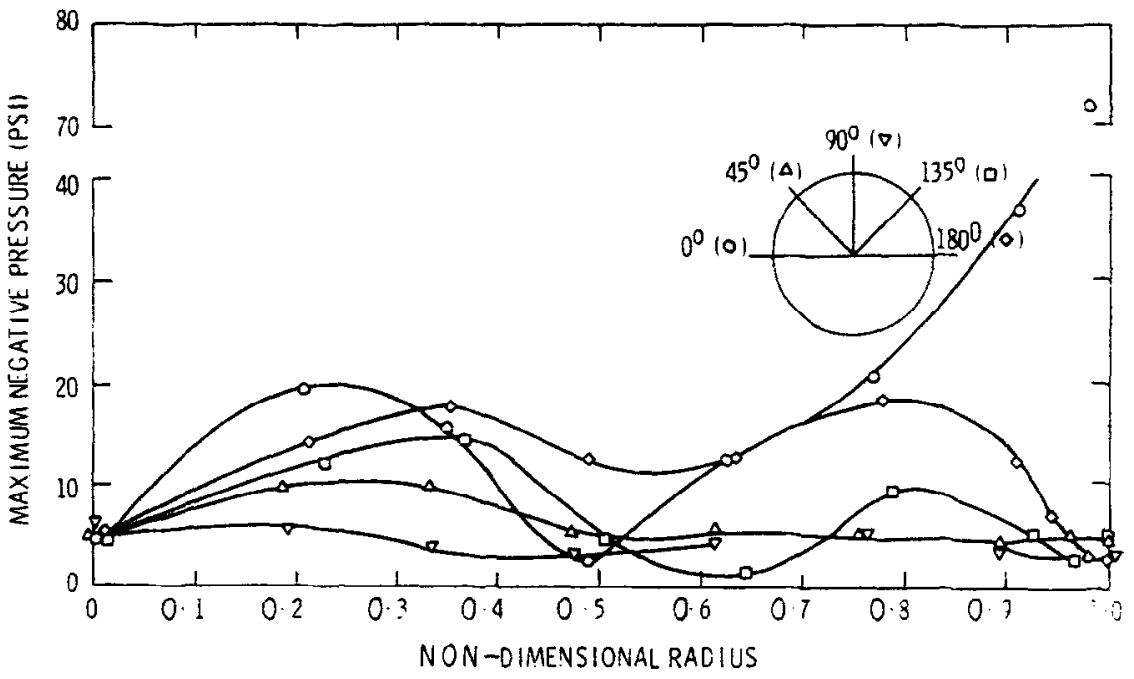

Fig. 7. Measured maximum negative pressure distribution in dilute polymer solution $(0.05$ per cent by weight of SEPARAN AP 30 in distilled water) subjected to a sinusoidal load over cone angle of $4 \cdot 6^{\circ}$ with onset of cavitation.

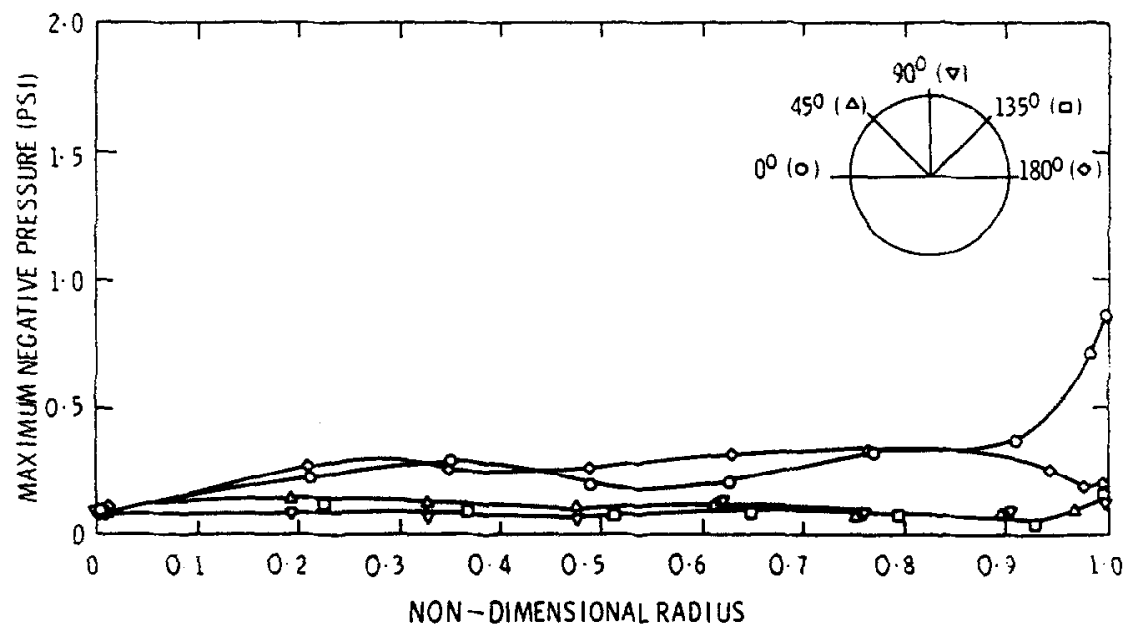

Fig. 8. Measured maximum negative pressure distribution in dilute polymer solution (0.05 per cent by weight of SEPARAN AP30 in distilled water) subjected to an impulsive load over cone angle of $4 \cdot 6^{\circ}$. 
water. The peak maximum negative pressure occurred near the input pole.

\section{Possible location of brain damage}

Treating the brain as an inviscid fluid, both the experimental results (for distilled water) and the Engin's model (Suh, 1971) predict that the maximum negative pressures resulting from a localized external load of sinusoidal type would occur in the brain at the locations illustrated by the encircled area $A$ in Fig. 9. However, an inpulsive type load would produce the maximum negative pressure at the location of impulse (area $B$ in Fig. 9) according to the experiments, while the analysis predicts the location at far inside as shown in Fig. 9 (area C). These predictions disagree with the experimental results obtained by Unterharnscheidt and Sellier (1966), which indicate the occurrance of the maximum negative pressure at the counter pole in the brain (area D in Fig. 9). Their results for an impact load show that the local pressure responses in the liquid including at the counter pole are in advance with the pressure responses at the pole. We are tempted to discredit their prediction based on the unreasonable results.

If the brain is considered a viscous fluid of $50 \mathrm{cP}$, the experimental data indicate the area $B$ in Fig. 9 to be the possible location of brain damage due to both the impulsive and periodic loads.

\section{CONCLUSIONS}

The purpose of this study is to investigate the response of axisymmetric, fluid-filled, thin elastic, closed spherical shell subjected

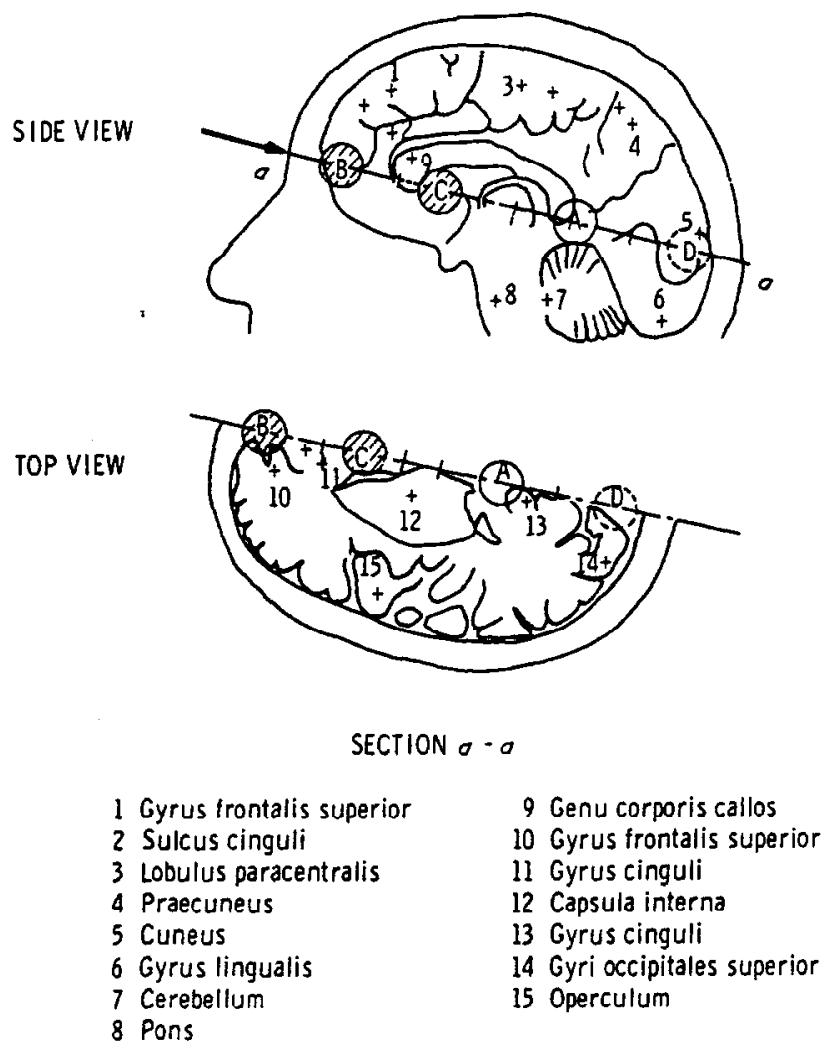

Fig. 9. Location of the maximum negative pressure in the brain resulting from localized external loads. 
to a localized, external load: of sinusoidal. square pulse, or impulsive nature. Experimentally an ultrasonic, sinusoidal input was directly imposed to the liquid at the interface between the shell and the liquid until the cavitation bubbles were observed in the liquid. By obtaining a pressure distribution at the onset of cavitation, the possible vulnerable regions were determined.

In the measurements for the distilled water under the sinusoidal input load, the peak negative pressure was found at the nondimensional radius of 0.32 on $180^{\circ}$ axis, at the onset of cavitation. Next peaks were near the horn tip and at the non-dimensional radius of 0.15 on the $0^{\circ}$ axis with slight difference in pressure. Numerical computations show that the peak negative pressure occurs at the nondimensional radius of 0.37 on the $180^{\circ}$ axis, and the next peak at the non-dimensional radius of 0.85 on the $0^{\circ}$ axis.

Both experimental and analytical studies (Engin. 1966; Benedict et al. 1970) on the cavitation pressure distribution in the inviscid fluid under the sinusoidal load indicate that the largest maximum negative pressure lie along the load axis within close neighborhoods. The analytical results for other loads such as an impulsive or square pulse load would give fairly good predictions with respect to vulnerable sites, provided the fluid is inviscid.

The experimental results for the dilute SEPARAN AP30 solution exhibited that the largest instantaneous negative pressure in response to both the sinusoidal and the square pulse inputs occur near the impact pole. The SEPARAN solution had the same viscosity of the brain, i.e. $50 \mathrm{cP}$.

\section{REFERENCES}

Benedict, J. V., Harris, E. H. and Von Rosenberg. D. U. (1970) An analytical investigation of the cavitation hypothesis of brain damage. J. bas. Engng 92, 597-603. Connolly, W. and Fox, F. E. (1954) Ultrasonic cavitation thresholds in water. J. acoust. Soc. Am. 26. 843-848.

Engin, A. E. (1969) The axisymmetric response of a fluid-filled spherical shell to a local radial impulse a model for heat injury. J. Biomechanics $2,325-341$.

Goldsmith. W. (1966) The physical processes producing head injury. In Proceedings of the Head Injury Conference, pp. 350-382. Lippincott.

Stalnaker, R. L. (1969) Mechanical properties of the head. Ph.D. Thesis, West Virginia University.

Suh, C. C. (1971) Cavitation in liquids contained in a spherical shell subjected to localized time-dependent external loads. Ph.D. Thesis, University of Michigan.

Unterharnscheidt, F. and Sellier, K. (1966) Mechanics and pathomorphology of closed brain injuries. In Proceedings of the Head Injury Conference, pp. 321-341. Lippincott. 